\title{
Méthode de relevé rapide de scènes urbaines tridimensionnelles dans le cadre d'évaluations environnementales stratégi- ques
}

\author{
Laplanche François * — Teller Jacques **_ Billen Roland *** \\ (*) Laboratoire SURFACES, Unité de Géomatique, Université de Liège, Allée du 6 Août, 17, \\ B5, 4000 Liège, Belgique \\ (**) Laboratoire d'Etudes Méthodologiques Architecturales (LEMA) Université de Liège \\ Chemin des Chevreuils, 1, B52, 4000 Liège, Belgique \\ (***) Service de Topographie et Géométrologie, Unité de Géomatique, Université de Liège \\ Allée du 6 Août, 17, B5, 4000 Liège, Belgique
}

f.laplanche@ulg.ac.be, jacques.teller@ulg.ac.be,rbillen@ulg.ac.be

\begin{abstract}
RÉSUMÉ. L'objectif du projet de recherche Européen SUIT (2001-2004) était de développer des méthodes d'évaluation de l'impact des projets ou de plans sur la conservation du patrimoine urbain dans le cadre de l'application des directives européennes en matière d'environnement (EIE et EES). Les différentes analyses appliquées aux fragments urbains nécessitent de disposer d'une modélisation tridimensionnelle de ceux-ci. Un complément de terrain est généralement nécessaire pour densifier le modèle, et pour ce faire, nous avons utilisé une méthode de photogrammétrie terrestre, la Direct Linear Transformation 3D (DLT 3D) pour sa facilité de mise en ouvre. Nous l'avons testée en l'appliquant à un fragment urbain de la ville de Liège et automatisée par la programmation de deux modules de calculs.

ABSTRACT. The aim of the European research project SUIT (2001-2004) was to develop methodologies for the impact assessment of projects or plans on the conservation of urban heritage in the framework of the application of European directives in environment (EIA and SEA). The different analyses on urban fragments require having $3 D$ model of it. A complementary survey is generally necessary to enhance the model and we use a terrestrial photogrammetric method called Direct Linear Transformation 3D (DLT 3D) for its easiness to use. We have tested it on an urban fragment of the city of Liège and automated it by the way of two computerized modules of calculation.
\end{abstract}

MOTS-CLÉS : EES, DLT 3D, SUIT, acquisition de données 3D urbaines, modèle 3D urbain.

KEYWORDS: SEA, DLT 3D, SUIT, Urban 3D data acquisition, 3D urban model.

Article publié dans la Revue Internationale de Géomatique. Volume 18(2) - $\mathrm{n}^{\circ} 2 / 2008$, pages 147 à 167 
Article publié dans la Revue Internationale de Géomatique. Volume 18(2) - n 2/2008, pages 147 à 167

\section{Introduction}

La directive européenne 85/337/CEE a établi en 1985 un premier cadre de référence, à l'échelon européen, pour l'évaluation des incidences sur l'environnement (EIE). Un de ses principaux objectifs était de réduire les disparités existant entre les États membres en matière de protection de l'environnement. On considérait, au début des années 1980, que de telles disparités constituaient une entrave à la concurrence et "affectaient ainsi le fonctionnement du marché commun ». Cette première directive a été amendée en 1997 (Directive 97/11/EC) et en 2003 (Directive 2003/35/EC), afin de préciser le champ d'application de l'EIE et de prendre en compte de nouvelles exigences en matière de participation et d'information des citoyens.

L'Evaluation Environnementale Stratégique (EES) consiste en l'élargissement formel de l'EIE aux politiques, plans et programmes. Elle a été définie comme :

"Le processus d'évaluation formalisé, systématique et complet des effets sur

l'environnement d'une politique, d'un plan ou d'un programme et de ses solutions alternatives, incluant la préparation d'un rapport écrit sur les résultats de cette évaluation et utilisant ces résultats dans une prise de décision publiquement responsable ». (Therivel et al., 1992)

L'EES a fait l'objet d'une nouvelle directive européenne, la Directive 2001/42/EC, portant sur l'évaluation des effets des plans et programmes liés à l'environnement. En effet, la Commission Européenne préconise aujourd'hui une approche hiérarchisée en matière d'évaluation des incidences environnementales. Il s'agit d'évaluer l'incidence de programmes ou de plans pour ensuite analyser l'incidence des projets qui s'inscrivent dans le cadre de ces plans ou programmes. Cette approche hiérarchisée s'explique par le fait que trop souvent des analyses de l'incidence de projets se voyaient vidées de leur sens par l'existence de plans qui cautionnaient ces projets de facto. D'où l'intérêt de faire remonter les analyses en amont, à un stade où les décisions sont encore ouvertes et susceptibles d'évoluer.

Ces deux procédures, l'EIE et l'EES, ont des répercussions importantes sur la conservation du patrimoine urbain, qu'il s'agisse de monuments et autres biens classés ou de centres historiques protégés, d'espaces publics symboliques du passé d'une ville ou encore, de points de vues remarquables sur le paysage urbain.

La Directive 2001/42/EC précise qu'une EES doit être réalisée lorsque les plans ou programmes concernés conditionnent des permis de bâtir, en particulier lorsque ceux-ci devraient être ultérieurement soumis à une EIE. Deux types de listes sont proposés dans la directive EIE pour décider de la nécessité ou non de soumettre un projet ou un plan à une étude d'incidence. L'annexe 1 de la directive est une liste fermée qui reprend les projets pour lesquels une étude d'incidence doit impérativement être réalisée, comme par exemple les installations nucléaires, les centrales d'épuration ou les voies rapides et autoroutes. L'annexe 2 de la Directive est une liste ouverte, qui reprend les projets pour lesquels une étude d'incidence 
devrait être envisagée dans certaines conditions. Les "travaux d'aménagement urbain, y compris la construction de centres commerciaux et de parkings" sont repris dans l'annexe 2. L'annexe 3 de la Directive précise les critères de sélections applicables aux projets repris dans l'annexe 2. Parmi ces critères, on retrouve d'abord des critères liés aux caractéristiques des projets, comme la dimension $d u$ projet ou le cumul avec d'autres projets devant être réalisés au même endroit. Les critères liés à la localisation comprennent la présence de "zones répertoriées ou protégées par la législation des États membres" ainsi que de "paysages importants du point de vue historique, culturel et archéologique". Les critères liés aux caractéristiques de l'impact potentiel reprennent bien entendu l'ampleur et l'étendue de l'impact, mais également la "réversibilité" de l'impact qui s'avère souvent un critère déterminant lorsque l'on s'intéresse au patrimoine.

Il s'ensuit qu'un grand nombre de projets et de plans d'aménagement sont désormais candidats à étude d'incidence, et ce en particulier lorsqu'ils portent sur des espaces situés dans un centre historique. Ce constat a motivé le développement du projet de recherche européen SUIT (2001- 2004), dont l'objectif était de développer des méthodes d'évaluation de l'impact de projets ou de plans sur la conservation du patrimoine urbain. Nous verrons à la section 2 que ces méthodes impliquent de disposer d'une modélisation tridimensionnelle de fragments urbains. La troisième section sera elle consacrée à la description des méthodes de relevé tridimensionnel existantes. Enfin, nous présenterons dans la section 4 une méthode originale d'acquisition des données 3D adaptée aux EIE et EES en milieu urbain densément construit.

\section{Le projet de recherche européen SUIT (2001-2004)}

Les procédures d'évaluation environnementales, telles que définies par les directives européennes EIE et EES, restent très ouvertes et flexibles. Le soin est laissé aux États membres et aux parties prenantes de donner vie à ce cadre général. Si l'on tient compte de l'étendue et de la complexité du sujet, on peut, à première vue, distinguer deux approches principales de la question. Une première approche consiste à traiter l'évaluation des incidences comme un processus de prise de décision. Il s'agit principalement de détailler et de comparer les différentes méthodologies (check-lists, scénarios, analyses multicritères) disponibles aux différentes étapes de cette procédure. Glasson et al. (1994) a présenté un relevé très détaillé de ce type. Une seconde approche se veut plus thématique et se concentre sur quelques points d'évaluation spécifiques. En elle-même, elle tend à être plus importante que la précédente, même si la nature procédurale de l'évaluation reste un aspect crucial. Les différents thèmes habituellement couverts vont de la protection de l'eau au bruit, en passant par la flore et l'analyse de l'impact social. Morris et Therivel (1995) ont rassemblé un échantillon représentatif de tels relevés thématiques, où figurait, de manière assez intéressante, un chapitre sur le paysage 
Article publié dans la Revue Internationale de Géomatique. Volume 18(2) - n 2/2008, pages 147 à 167

(Goodey, 1995) et un autre sur les atouts archéologiques et culturels ou matériels (Bourdillon et al., 1995).

Il convient de noter que les questions de patrimoine urbain ne sont pas largement couvertes par les références existantes en matière d'EIE et d'EES, même si, comme nous l'avons vu, il s'agit d'un aspect couvert par la directive européenne. Il s'agit pourtant d'un domaine tout à fait spécifique, tant en termes de procédure de prise de décision (irréversibilité, manque de connaissance préalable) qu'en matière de contenu d'évaluation (identification, valeur, incommensurabilité, etc.).

Le projet SUIT avait pour objectif de développer des outils et des méthodes qui permettent une meilleure prise en compte du patrimoine urbain dans les EIE et les EES. Ces méthodes portaient sur l'étape d'identification des projets et des plans à soumettre à étude d'incidence, sur le cadrage des études d'incidence (aspects à prendre en considération dans le cadre de l'analyse), sur l'analyse environnementale à proprement parler, ainsi que sur le suivi et l'amélioration ex post des projets et des plans. Nous nous attacherons plus particulièrement à l'analyse environnementale dans le cadre de cet article, dans la mesure où il s'agit d'une étape très gourmande en données tridimensionnelles. Cette étape se propose de prédire les effets qu'aura un bâtiment ou un plan dans le futur, de comparer les différentes alternatives envisagées lors du cadrage de l'étude et de proposer d'éventuelles mesures d'accompagnement, destinées à réduire les nuisances environnementales.

Les méthodes développées à cette fin dans le cadre du projet SUIT concernaient la visualisation de projets d'aménagement de fragments urbains (Tweed et al., 2003), l'analyse morphologique d'espaces publics (Teller, 2003) et les calculs en cycles de vie de l'évolution du stock bâti (Kohler, 2003). Une grille synthétique reprend ces différentes méthodes, les définit sommairement et identifie leur contexte d'application (Kohler et Bender, 2002).

Ces différentes méthodes impliquent de disposer d'une modélisation tridimensionnelle du milieu urbain. Traditionnellement les modèles utilisés portent sur le relief du site, les volumes des bâtiments, le mobilier urbain, le végétal ainsi que les éventuels tracés au sol (pavements, délimitation des voiries et des voies piétonnes). La modélisation des bâtiments porte sur le gabarit ainsi que les principaux décrochements de façades, ainsi que les portes et fenêtres ainsi que les principaux éléments saillants des toitures (cheminées, lucarnes etc.). Un exemple de modèle urbain est fourni à titre illustratif. Il s'agit de la place du vieux Marché à Prague, sur laquelle ont été réalisées des simulations de projets architecturaux alternatifs étudiés sur base de représentations visuelles stéréographiques (figure 1) ainsi que d'une analyse morphologique (figure 2). 

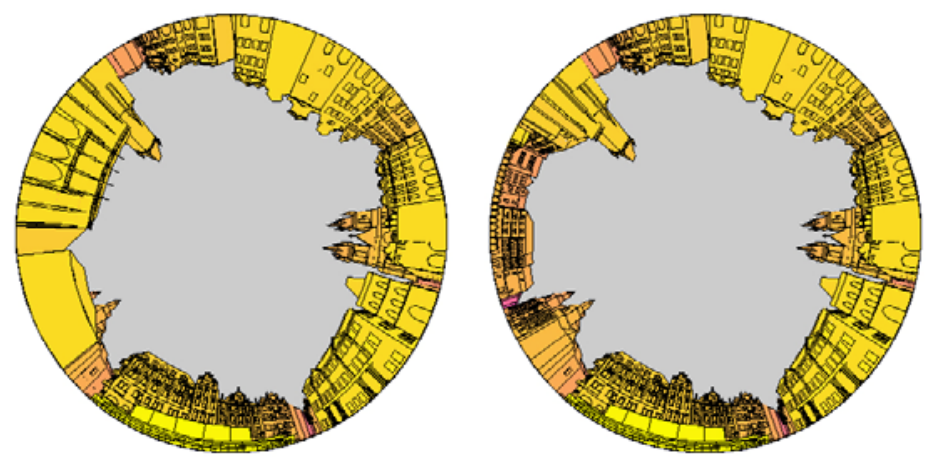

Figure 1. Projection stéréographique de la place de Old Town Square à Prague, avant et après destruction partielle de l'hôtel de ville en 1945 (à gauche de la figure)

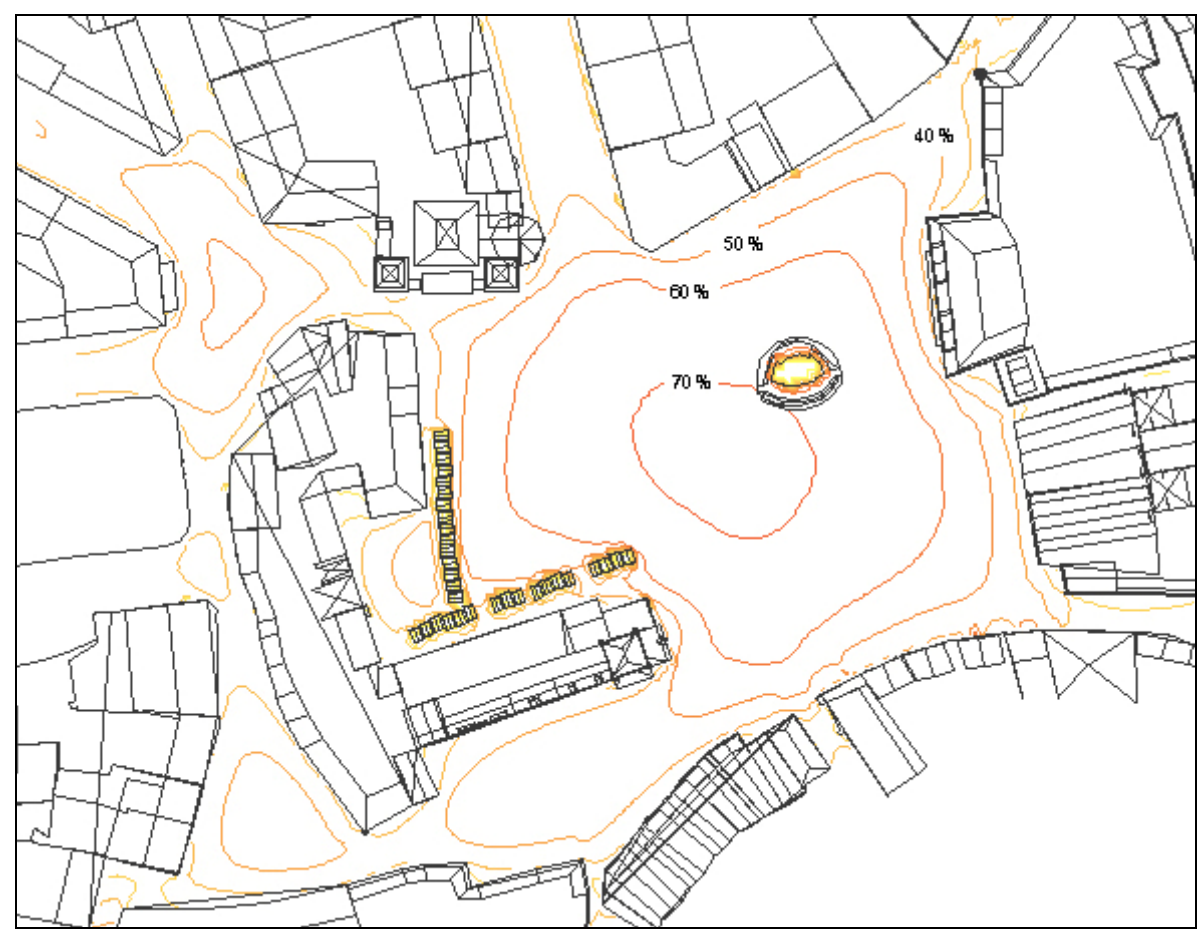

Figure 2. Analyse morphologique de la place de Old Town Square à Prague (ouvertures de ciel) après destruction de l'hôtel de ville en 1945. 
Article publié dans la Revue Internationale de Géomatique. Volume 18(2) - n 2/2008, pages 147 à 167

L'étendue spatiale des modèles porte d'ordinaire sur une dizaine à une vingtaine d'îlots, soit un rayon de quelques 250 mètres autour du projet ou de la place étudiée. Ce rayon dépend bien entendu de plusieurs facteurs. D'abord la morphologie générale du site et de la ville vont conditionner la taille du modèle. Pensons à titre d'exemple au centre historique de la ville de Metz, où une prise en compte de la silhouette de la ville implique de modéliser le relief aux alentours. L'étendue du modèle dépend également de la nature de l'intervention projetée. Elle devra être d'autant plus large que le bâtiment est imposant. Elle sera en général plus importante pour un plan que pour un projet.

Enfin, il n'est pas inutile de rappeler que les délais d'usage dans le cadre de la réalisation d'EIE et EES sont d'ordinaire très contraignants. Ces délais vont bien entendu varier entre les Etats Membres et d'une étude à l'autre, mais on considère que la durée moyenne de réalisation d'une étude d'incidence est de l'ordre de 2 à 3 mois, entre le moment où l'on commence l'analyse et l'échéance de dépôt du dossier complet par les auteurs de l'étude d'incidence (Land Use Consultants, 1996). Ce délai moyen est un peu plus long pour les EES, mais les modèles et les analyses sont dans ce cas encore beaucoup plus lourds. Il est bien entendu que, dans ce contexte, l'acquisition de l'information tridimensionnelle ne peut en général prendre plus de deux à trois semaines, ce qui va constituer un paramètre critique dans le cadre de la comparaison des différentes solutions techniques disponibles à l'heure actuelle.

\section{Les méthodes de relevé tridimensionnel}

Dans la plupart des cas, on ne dispose pas a priori d'une modélisation tridimensionnelle complète de la zone à étudier. Par contre, des données utilisables, même partielles, existent souvent. L'expert sera donc confronté à des données d'origines diverses qu'il devra intégrer et compléter. Ainsi, au-delà du choix d'une méthode de relevé répondant aux contraintes inhérentes aux EES, l'expert doit pouvoir apprécier la qualité et l'adéquation de données tridimensionnelles et cela quels que soient leurs modes d'acquisition.

Ces modes d'acquisition de données tridimensionnelles sont nombreux, variés et en constante évolution. Même en se limitant à l'information relative à la surface des objets urbains (sans prendre en compte les objets souterrains et l'intérieur des bâtiments), la diversité des méthodes offertes est impressionnante. Loin de vouloir dresser un état de l'art exhaustif sur le sujet, nous présentons une classification très générale de ces modes d'acquisition nous permettant notamment de mettre en exergue l'intérêt de notre approche. De ce fait, les références proposées dans cette section sont illustratives et n'ont pas la prétention de couvrir l'ensemble des recherches en cours. 
Les modes d'acquisition de données urbaines tridimensionnelles peuvent se diviser en deux catégories suivant que l'acquisition se fasse par voie aérienne ou voie terrestre. La première catégorie inclut les levés photogrammétriques et les levés lasérométriques.

Traditionnellement, la photogrammétrie aérienne est un grand pourvoyeur de données tridimensionnelles. Une partie importante des données spatiales récoltées par les instituts cartographiques le sont par restitution de couples stéréoscopiques de photographies aériennes (argentiques ou numériques) (figure 3) (PICC, 2004). Les recherches actuelles en photogrammétrie digitale concernent notamment l'extraction automatique d'objets (bâtiments, routes, etc.) (Kim et al., 2001 ; Fuchs et al., 2002). Citons également l'utilisation d'autres vecteurs spatiaux pour l'obtention de données sur des zones plus restreintes, notamment l'emploi d'hélicoptères (Gruen et al., 2003). Avec l'avènement des satellites de très haute résolution, la photogrammétrie satellitaire a fait son entrée dans le domaine de l'acquisition de données urbaines tridimensionnelles (Chehata, 2005). Même si le niveau de détail (résolution géométrique) des objets relevés est moindre qu'en utilisant des photographies aériennes, ce type de senseurs constitue une véritable alternative.

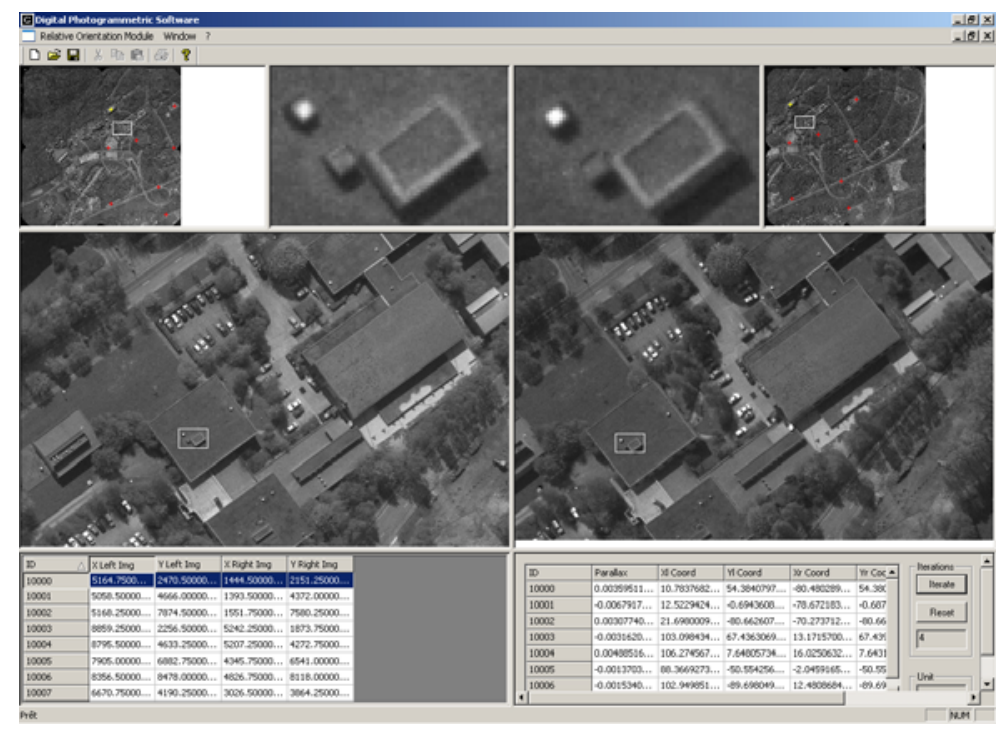

Figure 3. DDPS, un logiciel de photogrammétrie digitale (Schumacker, 2002).

Depuis une quinzaine d'années, l'utilisation de laser aéroporté est passée du stade expérimental au stade de production à grande échelle (Wiechert, 2004). Citons essentiellement les systèmes actifs LIDAR (Laser-Induced Detection And Ranging) pourvoyeurs de données pour les applications urbaines (figure 4). Ces systèmes 
Article publié dans la Revue Internationale de Géomatique. Volume 18(2) - n 2/2008, pages 147 à 167

peuvent être combinés avec d'autres senseurs (photographies, scanneurs multispectraux, etc.) élargissant la gamme des produits accessibles (Rottensteiner, 2003). Notons finalement que l'ensemble des techniques précitées ont grandement bénéficié du développement des systèmes GPS inertiels qui permettent une connaissance précise de la position et de l'orientation des différents vecteurs spatiaux. Une comparaison plus approfondie de ces techniques aériennes et satellitaires peut être trouvée notamment dans (Balastavias et Gruen, 2003).

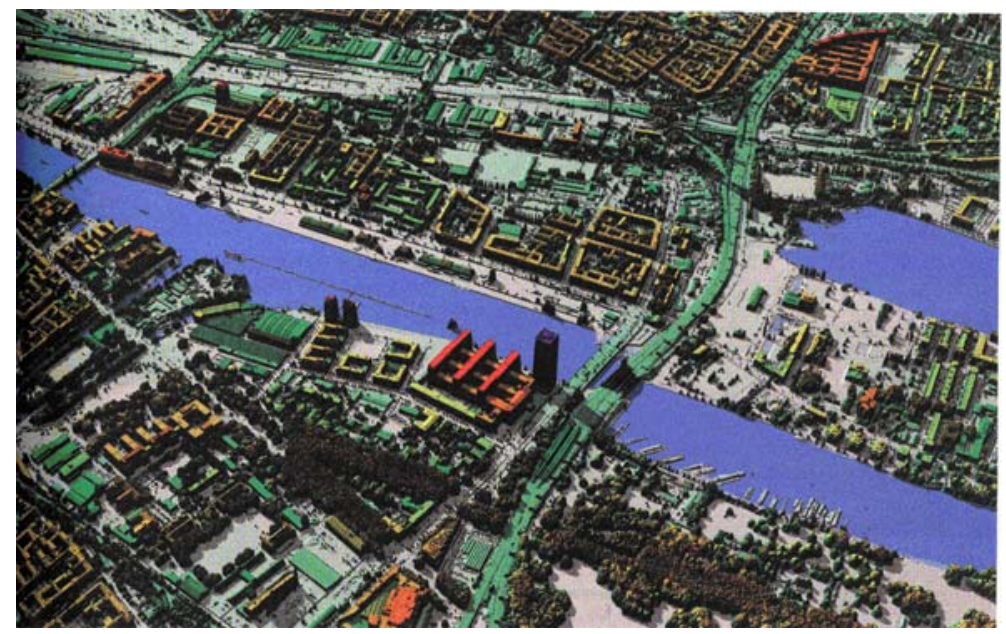

Figure 4. Modèle 3D de la ville de Berlin. (Wiechert, 2004)

Les méthodes « terrestres » d'acquisition de données sont également nombreuses et diverses. La première des techniques présentées est un peu particulière car elle ne concerne que la détermination de la hauteur d'objets d'emprise connue. La démarche est simple et assez souvent utilisée car flexible et peu coûteuse. Connaissant l'emprise d'un bâtiment, il suffit d'obtenir une information sur la hauteur de celui-ci et considérer l'objet tridimensionnel correspondant comme l'extrusion de l'emprise par la hauteur. Evidemment, on obtient un objet simplifié, plus ou moins généralisé par rapport à la forme réelle du bâtiment. La mesure de la hauteur peut se faire de diverses manières : - estimation sur base d'une comparaison avec un élément de référence (nombre d'étage, mire, etc.) ; - relevé par station totale (distancemètre, laser, etc.). Notons que cette démarche est souvent utilisée en combinaison avec des données obtenues par voie aérienne (notamment LIDAR), dans ce cas la forme du bâtiment peut être obtenue avec plus de précision. Au-delà de ce cas particulier, plusieurs méthodes permettent d'obtenir un relevé tridimensionnel complet par voie terrestre: relevé par station totale, relevé photogrammétrique, relevé laser. 
Relever un bâtiment à l'aide d'une station totale a toujours été techniquement possible mais les dernières évolutions des distancemètres électroniques (laser) permettant des prises de mesures de distances sans réflecteurs augmentent considérablement son utilisation. La photogrammétrie terrestre est quant à elle depuis longtemps utilisée pour des relevés de façades et par extension pour le relevé de données 3D du milieu urbain. L'utilisation de chambres calibrées suivant les techniques traditionnelles est quelque peu fastidieuse par rapport à une utilisation plus simple d'appareil numérique non calibré (Zhao et Shibasaki, 2001a). Finalement, la dernière (r)évolution concernant la prise de mesure 3D est sans nul doute les scanneurs laser terrestres (Haala, 2004), les systèmes les plus répandus étant les systèmes par balayage. Ces diverses techniques présentent l'avantage de la non occupation des points à relever et partagent également le problème de la reconstitution d'un modèle tridimensionnel unique et complet sur base de différentes prises de mesures ou de clichés. Notons finalement dans le cas de la photogrammétrie et du laser que les recherches actuelles explorent l'utilisation de vecteurs mobiles (voitures, robots, etc.) afin d'accélérer la collecte de l'information (Zhao et Shibasaki, 2001b).

Au-delà de cette classification, l'obtention de données urbaines tridimensionnelles est plus souvent le fruit de combinaisons des méthodes exposées : combinaisons de données de sources aériennes (Rottensteiner et Jansa, 2002), de sources terrestres (Stamos et Allen, 2000) ou bien des deux (Früh et Zakhor, 2003). Par exemple, Haala et Brenner (1999) combinent l'utilisation d'une carte en 2D pour le positionnement des objets en planimétrie et les valeurs d'altitude d'un modèle numérique de surfaces obtenu par un scanner laser afin de générer un MNS. Après simplification, le MNS présente une allure proche de la forme des bâtiments et les textures des façades sont obtenues par rectification de photographies prise par voies terrestres.

Une première analyse des ces différentes méthodes sous le filtre des contraintes inhérentes aux EES nous permet de tirer les enseignements suivants :

- D'une manière générale, les méthodes «aériennes » sont bien adaptées à l'acquisition de données sur des zones relativement étendues, pour lesquelles l'obtention de surface de référence (MNS, MNT) est un objectif important. Le coût bien que variable en fonction de la méthode retenue, est assez important voir prohibitif dans le cas de relevé d'extension restreinte. Le temps de traitement de l'information est généralement non négligeable et s'accommode mal de mise en œuvre rapide et de délais de livraison courts. De plus, leur niveau de détail est généralement insuffisant pour réaliser des évaluations à partir de l'espace public et ne convient pas directement pour analyser l'impact de différentes alternatives sur la perception du patrimoine bâti.

- D’une manière générale, les méthodes terrestres permettent de beaucoup mieux appréhender les spécificités architecturales des centres urbains. 
Article publié dans la Revue Internationale de Géomatique. Volume 18(2) - n 2/2008, pages 147 à 167

Néanmoins, la complexité de la mise en œuvre des techniques ainsi que leurs coûts sont assez variables. Outre son coût d'utilisation, le scanneur laser a pour limitation actuelle le temps relativement important de traitement de l'information (proportionnelle à la richesse des éléments à extraire). La mise en œuvre des techniques photogrammétriques classiques (chambre métrique) reste lourde, tandis que le levé à la station totale peut s'avérer fastidieux. Comme nous le détaillons par la suite, l'utilisation de techniques photogrammétries digitales légères semble être la plus adéquate.

\section{Une méthode pratique de relevés adaptée aux EES en milieu urbain}

Une des premières tâches de la procédure d'étude d'incidence est de récolter et intégrer les données tridimensionnelles existantes. Dans la plupart des cas, un complément de terrain pour densifier ou mettre à jour ces données de base sera nécessaire et l'utilisation d'une méthode légère, facile à mettre en place en un temps très court s'avérera bien souvent indispensable. Dans les études qui nous intéressent, une précision de l'ordre de 10 à 20 centimètres nous est apparue comme suffisante (Laplanche, 2004). Ce qui est essentiel ici, c'est la facilité d'utilisation de la méthode et l'obtention rapide des coordonnées tridimensionnelles.

$\mathrm{Au}$ vu de la précision désirée, la photogrammétrie terrestre à partir de photographie numérique s'est très vite imposée comme une solution très séduisante pour notre problème.

Néanmoins, plusieurs contraintes nous étaient imposées. D'une part, les utilisateurs finaux de l'application ne possèdent aucune connaissance en photogrammétrie. D'autre part, tous les paramètres de l'appareil sont susceptibles de varier: zoom, résolution... L'utilisation de méthodes photogrammétriques classiques avec orientation interne était donc difficilement envisageable (Drap et Grussenmeyer, 2005). Les logiciels actuels, comme Photomodeler par exemple (Photomodeler, 2005), ne sont pas non plus applicables puisque leur utilisation suppose l'emploi d'un même appareil, sans modification de zoom. En outre, nous désirons également maîtriser l'ensemble des paramètres de la méthode utilisée. Ce travail s'inscrivant dans une logique de recherche à plus long terme, le module développé s'intègrera au logiciel de photogrammétrie développé au sein de notre université (Da Col et al., 2002 ; Schumacker, 2002) et des modules supplémentaires sont susceptibles d'être développés.

La méthode Direct Linear Transformation (DLT) 3D (Kwon, 1998) est apparue comme un choix judicieux car elle est très souple à mettre en œuvre et elle correspond à nos exigences en matière de précision. Ses fondements théoriques sont rappelés au point suivant. La mise en oeuvre de la méthode a été testée sur un fragment urbain relativement dense, la place du Marché de Liège. Nous détaillerons 
les étapes de cette mise en œuvre. Enfin, le traitement des résultats et son automatisation par la programmation de deux modules de calculs seront présentés.

\subsection{Aspects théoriques de la DLT $3 D$}

La DLT 3D est une méthode de photogrammétrie terrestre qui permet à partir d'un minimum de 2 clichés et de 6 points d'appui de déterminer les coordonnées tridimensionnelles de l'ensemble des points communs aux deux clichés. Si l'on désire augmenter la précision, on peut augmenter le nombre de clichés ou le nombre de points d'appui, voire les deux simultanément. On peut également choisir de prendre en compte les erreurs optiques de la lentille de l'appareil utilisé.

La première étape de la méthode consiste à réaliser la calibration de l'ensemble des clichés chacun pris séparément à l'aide d'une série de paramètres. Ces paramètres sont au nombre de 11 à 16 selon qu'on désire tenir compte ou non de la distorsion de la lentille (voir tableau 1). A chaque cliché, est donc associée une série de paramètres qui lui sont propres et qui permettent de l'orienter. Contrairement aux méthodes classiques de photogrammétrie, on réalise d'abord une orientation absolue des clichés en calculant la position spatiale de la gerbe perspective à partir de coordonnées terrain. Les points d'appui des différents clichés sont soit différents, soit les mêmes si on désire en limiter le nombre, mais l'orientation de chaque cliché reste indépendante.

Pour chacun des points d'appui connus en $\mathrm{X}, \mathrm{Y}$ et $\mathrm{Z}$, la méthode donne la relation suivante entre les coordonnées terrain et les coordonnées image $(\mathrm{u}, \mathrm{v})$ :

$$
\begin{gathered}
\frac{1}{R} u=\frac{1}{R}\left(L_{1} x+L_{2} y+L_{3} z+L_{4}-L_{9} u x-L_{10} u y-L_{11} u z\right)+\Delta u \\
\frac{1}{R} v=\frac{1}{R}\left(L_{5} x+L_{6} y+L_{7} z+L_{8}-L_{9} v x-L_{10} v y-L_{11} v z\right)+\Delta v \\
\text { où } \\
R=L_{9} x+L_{10} y+L_{11} z+1 \\
\Delta u=\xi\left(L_{12} r^{2}+L_{13} r^{4}+L_{14} r^{6}\right)+L_{15}\left(r^{2}+2 \xi^{2}\right)+L_{16} \xi \eta \\
\Delta v=\eta\left(L_{12} r^{2}+L_{13} r^{4}+L_{14} r^{6}\right)+L_{15} \xi \eta+L_{16}\left(r^{2}+2 \eta^{2}\right)
\end{gathered}
$$

avec

$$
\begin{array}{ll}
{[\xi, \eta]=\left[u-u_{0}, v-v_{0}\right]} \\
r^{2}=\xi^{2}+\eta^{2}
\end{array} \text { et } \begin{aligned}
& u_{0}=\frac{L_{1} L_{9}+L_{2} L_{10}+L_{3} L_{11}}{L_{9}{ }^{2}+L_{10}{ }^{2}+L_{11}{ }^{2}} \\
& v_{0}=\frac{L_{5} L_{9}+L_{6} L_{10}+L_{7} L_{11}}{L_{9}{ }^{2}+L_{10}{ }^{2}+L_{11}{ }^{2}}
\end{aligned}
$$


Article publié dans la Revue Internationale de Géomatique. Volume 18(2) - n 2/2008, pages 147 à 167

Comme à la base les paramètres $\mathrm{L}_{9}, \mathrm{~L}_{10}$ et $\mathrm{L}_{11}$ ne sont pas connus, il faut travailler par itérations successives. Une bonne valeur de départ pour converger rapidement est simplement zéro. Notons que, si on ne désire pas prendre en compte les erreurs optiques, $\Delta \mathrm{u}$ et $\Delta \mathrm{v}$ sont nuls et les paramètres $\mathrm{L}_{12}$ à $\mathrm{L}_{16}$ ne sont pas calculés. Dans le cas contraire, on a besoin de connaître les coordonnées du point principal $\left(\mathrm{u}_{0}, \mathrm{v}_{0}\right)$. Celles-ci sont également obtenues par itérations successives. Le centre de l'image a été utilisé comme valeur lors de la première itération. Ces équations peuvent être mises sous la forme matricielle, et le système peut être résolu directement ou par moindres carrés selon le nombre de points d'appui considérés, pour connaître les valeurs les plus probables des paramètres $\mathrm{L}_{1}$ à $\mathrm{L}_{16}$. Les points d'appui connus en $\mathrm{X}, \mathrm{Y}$ et $\mathrm{Z}$ ne peuvent être coplanaires. En outre, le nombre minimum de points d'appui varie selon le nombre de paramètres qu'on désire prendre en compte (voir tableau 2).

\begin{tabular}{|l|l|}
\hline Paramètres & Remarques \\
\hline$L_{1}-L_{11}$ & paramètres standard de la DLT \\
\hline$L_{12}-L_{14}$ & Termes de distorsion optique du $3^{\text {ème }}, 5^{\text {ème }}$ et $7^{\text {ème }}$ ordre \\
\hline$L_{15} \& L_{16}$ & Termes de distorsion de décentrement \\
\hline
\end{tabular}

Tableau 1. Les paramètres de calibration des clichés de la méthode DLT 3D. (d’après Kwon, 1998)

\begin{tabular}{|c|c|}
\hline Nombre. de paramètres & Nombre minimum de points d'appui \\
\hline 11 & 6 \\
\hline 12 & 6 \\
\hline 14 & 7 \\
\hline 16 & 8 \\
\hline
\end{tabular}

Tableau 2. Nombre minimum de points d'appui par rapport au nombre de paramètres utilisés. (d'après Kwon, 1998)

Une fois les différents clichés calibrés, on peut procéder à la reconstruction, c'est-à-dire au calcul des coordonnées tridimensionnelles d'un point identifiable sur l'ensemble des clichés utilisés. On réalise donc en quelque sorte une orientation relative a posteriori. Pour chaque cliché, on a : 


$$
\begin{gathered}
\frac{1}{R}\left(v L_{9}-L_{1}\right) x+\frac{1}{R}\left(v L_{10}-L_{2}\right) y+\frac{1}{R}\left(v L_{11}-L_{3}\right) z=\frac{1}{R}\left(L_{4}-v\right) \\
\frac{1}{R}\left(\omega L_{9}-L_{5}\right) x+\frac{1}{R}\left(\omega L_{10}-L_{6}\right) y+\frac{1}{R}\left(\omega L_{11}-L_{7}\right) z=\frac{1}{R}\left(L_{8}-\omega\right) \\
\text { où } \\
{[v, \omega]=[u-\Delta u, v-\Delta v]} \\
R=L_{9}^{(i)} x+L_{10}^{(i)} y+L_{11}^{(i)} z+1
\end{gathered}
$$

Ici, on connaît, pour chaque cliché, les paramètres $\mathrm{L}_{1}$ à $\mathrm{L}_{16}\left(\mathrm{ou} \mathrm{L}_{11}\right)$ ainsi que les coordonnées image du point, et $\mathrm{x}, \mathrm{y}$ et $\mathrm{z}$ sont les inconnues. De nouveau, comme $\mathrm{x}$, $\mathrm{y}$ et $\mathrm{z}$ interviennent dans le calcul de $\mathrm{R}$, on devra travailler par itérations successives. Ce système se résout soit directement, soit par moindres carrés selon le nombre de clichés pris en compte.

\subsection{Mise en œuvre de la DLT 3D sur un site urbain}

Notre cas d'étude était la place du Marché de Liège. Nous avons testé l'utilisation de la DLT 3D à partir de soit deux, soit trois clichés d'une même séquence de bâtiments ainsi qu'avec dix points d'appui.

Les clichés ont été pris avec un appareil photographique numérique possédant une résolution de 4,5 mégapixels. Pour plus de stabilité, l'appareil était fixé sur un pied lors des prises de vue. Pour limiter la parallaxe, nous avons évité de prendre des clichés sous un angle trop rasant. Un angle minimum d'environ 30 degrés entre l'axe optique et l'alignement des façades a été respecté.

Pour mesurer les points d'appui, nous avons utilisé une station totale avec visée laser. La portée du laser était de 50 mètres.

Le travail sur site s'est effectué en deux fois, tout d'abord, les prises de vue avec l'appareil photo numérique, ensuite, le levé des points d'appui à la station totale. La réalisation de ces deux tâches à deux moments distincts, nous a permis, pour optimaliser le levé, d'imprimer les différents clichés et d'y repérer les points communs aux clichés d'une même séquence pour ainsi limiter le nombre de points d'appui à lever. Les points étaient distribués sur l'ensemble de la façade. En outre, nous avons pris des points en saillie et en retrait pour assurer une non coplanarité de ceux-ci. Comme le but du travail était de valider une méthode de relevé tridimensionnel et non d'effectuer un levé complet de la place, nous n'avons sélectionné que quelques séquences de bâtiments.

Pour permettre une validation de la méthode, un nombre plus important de point a été levé. En pratique, entre 15 et 20 points ont été levés pour chaque séquence de bâtiments. 
Article publié dans la Revue Internationale de Géomatique. Volume 18(2) - n 2/2008, pages 147 à 167

\subsection{Calculs et résultats}

Deux modules spécifiques pour le calcul des coordonnées tridimensionnelles des points communs à deux ou trois clichés orientés grâce à dix points de calage ont été programmés en Visual Basic.

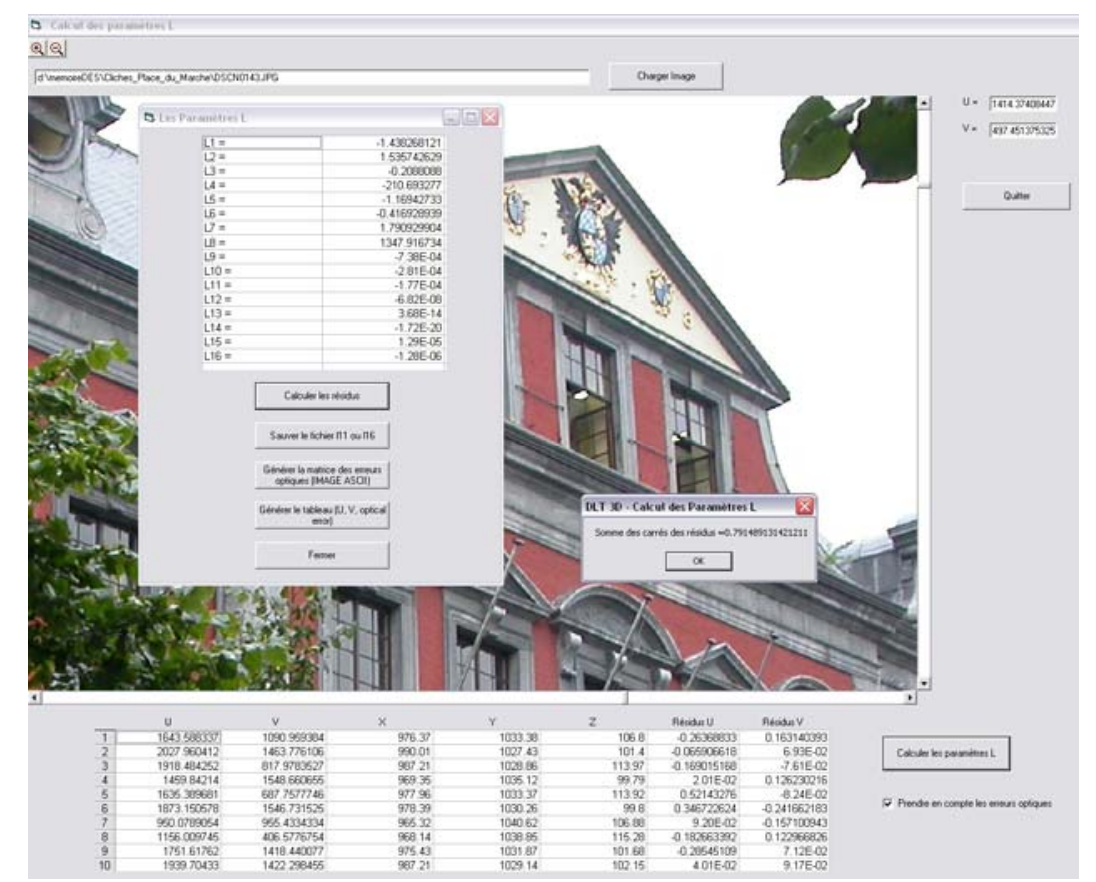

Figure 5. L’interface du module 1 : calcul des paramètres $L$.

Le premier module permet, à partir de dix points d'appui de calculer les paramètres d'orientation L de n'importe quel cliché. Selon que l'on désire ou non prendre en compte les erreurs optiques, 11 ou 16 paramètres seront calculés. La figure 5 ci-dessus présente l'interface de ce module. Elle est constituée d'une fenêtre comprenant le cliché dans lequel on peut se déplacer grâce à des barres de navigation et à un outil de zoom. Deux zones de texte, dans le coin supérieur droit, nous renseignent sur les coordonnées image du curseur, et lorsqu'on clique, les coordonnées image sont écrites dans un tableau situé dans la partie inférieure de l'interface. Lorsque les dix points d'appui ont été pointés dans l'image, les coordonnées réelles tridimensionnelles de ces points doivent être saisies au clavier dans les cases du tableau prévues à cet effet. Après cela, le calcul des paramètres L peut être lancé. Une nouvelle fenêtre s'ouvre lorsque le calcul est résolu. Celle-ci offre la possibilité de calculer les résidus sur les points d'appui pour valider l'ajustement, ensuite, si ces derniers sont acceptables, les 11 ou 16 paramètres 
d'orientation peuvent être enregistrés dans un fichier texte de même nom que le fichier image et ayant comme extension *.111 ou *.116 (Analogie avec le principe des fichiers de géoréférenciation d'image sous ArcView : *.tfw). Lorsque les erreurs optiques sont prises en comptes, une image de la modélisation de ces erreurs par la méthode de la DLT 3D peut être générée. A chaque pixel de cet image est associée la valeur $\sqrt{\Delta u^{2}+\Delta v^{2}}$ (déplacement en pixels du à la prise en compte des erreurs optiques - voir figure 7).

Le second module permet le calcul proprement dit des coordonnées $\mathrm{X}$, $\mathrm{Y}$, et Z de tout point commun aux différents clichés d'une même scène. Les images, préalablement orientées grâce au module 1 sont chargées dans l'interface et les points dont on désire connaitre les coordonnées sont pointés dans les deux ou trois clichés chargés. Une fois les points sélectionnés, le calcul des coordonnées tridimensionnelles est effectué. Ces coordonnées peuvent ensuite être exportées vers un fichier texte aisément récupérable dans un logiciel de CAO. La figure 6 cidessous présente l'interface du module 2 .

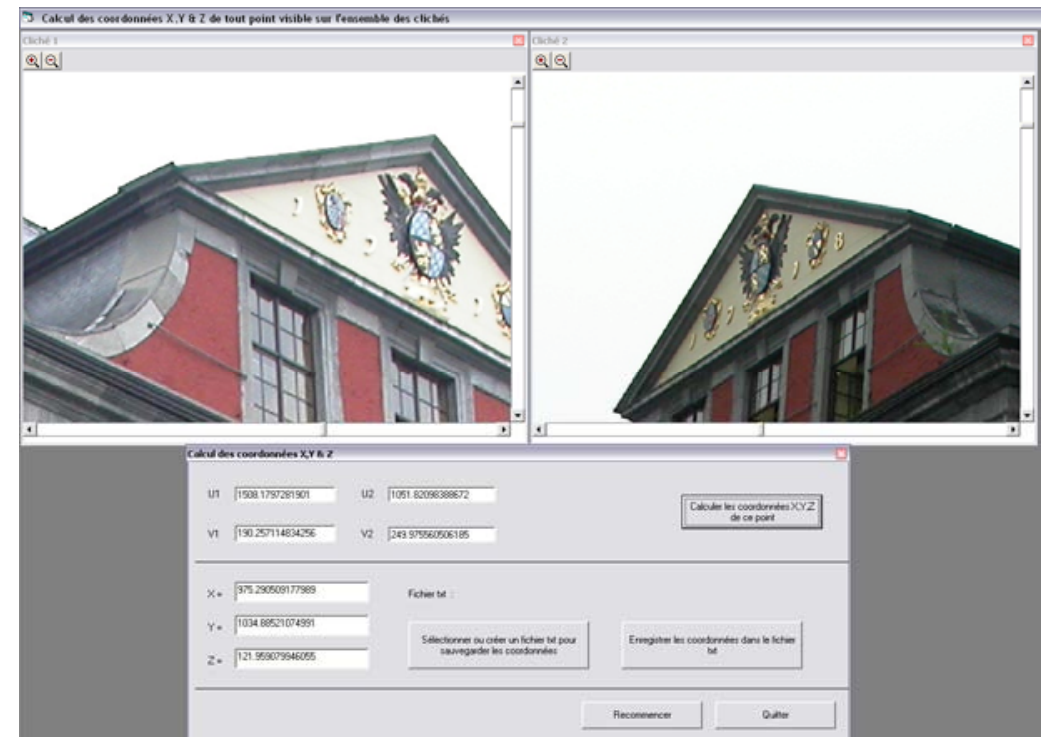

Figure 6. L'interface du module 2 : le calcul des coordonnées $X, Y$ et $Z$.

Grâce à ces deux modules, il est aisé de récupérer les coordonnées tridimensionnelles d'une scène urbaine. 
Article publié dans la Revue Internationale de Géomatique. Volume 18(2) - n 2/2008, pages 147 à 167

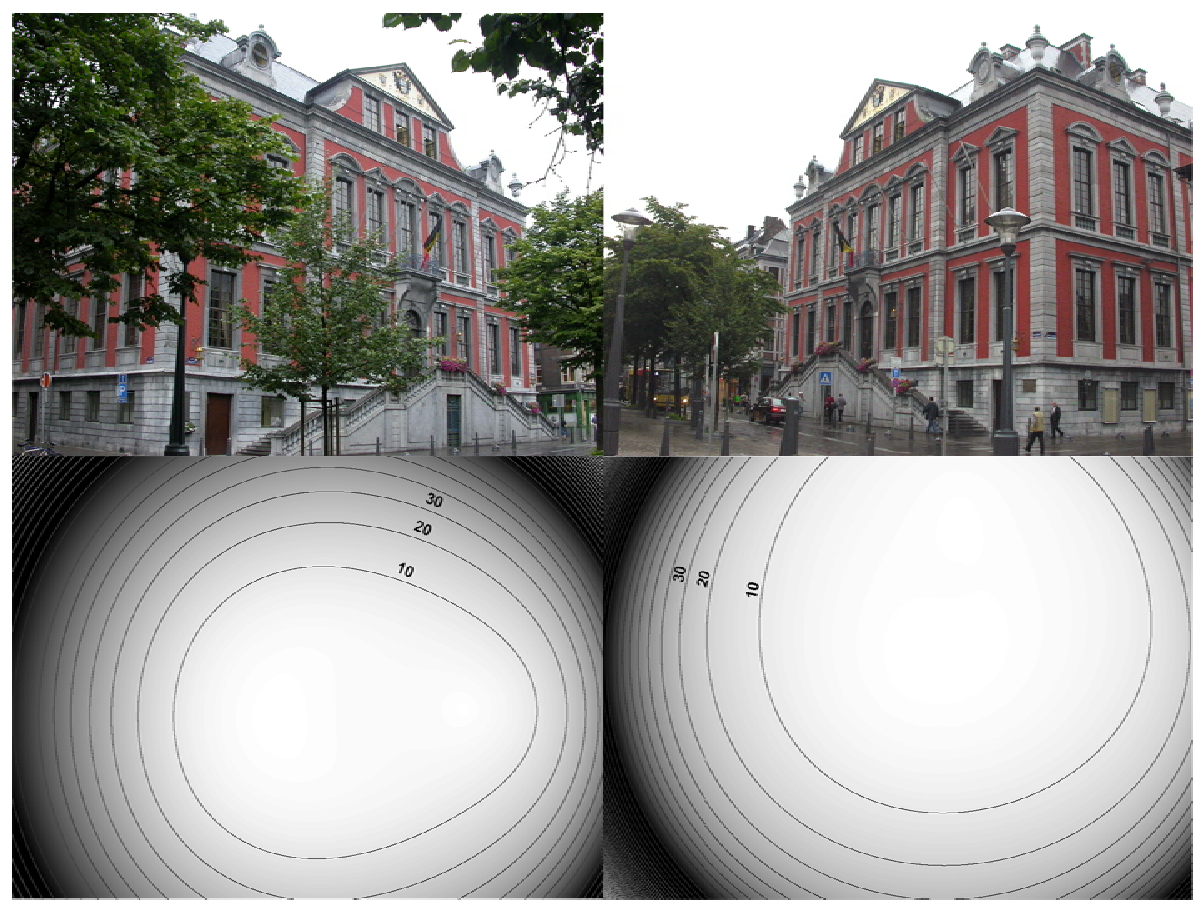

Figure 7. Les clichés de l'Hôtel de Ville et la modélisation des erreurs optiques par la méthode de la DLT $3 D$.

\begin{tabular}{|c|c|c|c|c|c|c|}
\hline \multirow{2}{*}{ Points } & \multicolumn{2}{|c|}{ Résidus avec 16 paramètres } & \multicolumn{3}{c|}{ Résidus avec 11 paramètres } \\
\cline { 2 - 7 } & DX & DY & DZ & DX & DY & DZ \\
\hline SL 1014 & -0.01 & 0.00 & 0.00 & 0.01 & 0.04 & 0.04 \\
\hline SL 1015 & 0.00 & 0.00 & 0.00 & 0.00 & -0.04 & -0.02 \\
\hline SL 1018 & 0.00 & 0.00 & 0.00 & 0.03 & 0.01 & 0.00 \\
\hline SL 1023 & 0.00 & 0.00 & 0.00 & -0.06 & -0.01 & 0.00 \\
\hline SL 1020 & 0.00 & -0.01 & 0.00 & 0.00 & -0.03 & -0.03 \\
\hline SL 1022 & 0.01 & 0.00 & 0.00 & 0.07 & -0.03 & -0.02 \\
\hline SL 2102 & 0.00 & 0.00 & 0.00 & 0.09 & 0.03 & 0.00 \\
\hline SL 2103 & 0.00 & 0.00 & 0.00 & -0.07 & -0.02 & -0.02 \\
\hline SL 2105 & -0.01 & 0.00 & 0.00 & -0.02 & 0.01 & 0.02 \\
\hline SL 2106 & 0.00 & 0.00 & 0.00 & -0.03 & 0.04 & 0.03 \\
\hline SL.1016 & -0.02 & 0.01 & 0.02 & -0.03 & -0.01 & 0.00 \\
\hline SL.1017 & -0.04 & 0.02 & 0.02 & -0.11 & -0.07 & -0.01 \\
\hline SL.1019 & 0.00 & -0.02 & -0.02 & -0.01 & -0.01 & -0.04 \\
\hline SL.2104 & 0.02 & -0.02 & 0.00 & -0.03 & -0.01 & 0.03 \\
\hline 3000 & -0.01 & 0.00 & 0.01 & 0.01 & 0.03 & 0.04 \\
\hline
\end{tabular}

Tableau 3. Résidus calculés sur quelques points de contrôle après ajustement des clichés avec 11 ou 16 paramètres (en gris, les points d'appui). 
Pour vérifier la qualité de l'ajustement, nous avons calculé les résidus d'un certain nombre de points de contrôle (Tableau 3). Parmi ceux-ci, nous avons recalculé les coordonnées des points d'appui qui ont servi aux calculs des paramètres L d'orientation des clichés. Dans une méthode photogrammétrique classique, ceci n'aurait pas beaucoup de sens puisque ces points sont bons par construction, mais dans le cas de la DLT 3D, les calibrations de chaque cliché étant indépendantes, les résidus sur un même point d'appui calculés dans plusieurs clichés ne sont pas corrélés. Dès lors, même si l'on peut s'attendre à ce que les résidus sur ces points soient légèrement inférieurs à ceux des autres points, ils renseignent tout de même, selon nous, sur la précision de la méthode.

On observe que la prise en compte des erreurs optiques lors de l'ajustement, améliore de manière non négligeable la qualité. La figure 7 ci-dessus illustre la façon dont le DLT 3D modélise ces erreurs optiques. On y observe que l'ensemble de la façade commune aux deux clichés est concentrée sous l'isoligne 30 (déplacement de 30 pixels) ce qui peut expliquer le fait que les résidus calculés sans prise en compte des erreurs optiques restent faibles. Dans tous les cas, les résidus obtenus sont bien inférieurs aux dix centimètres que nous avions préconisés. Les autres séquences de bâtiments levées présentent des résultats du même ordre de grandeur que ceux présentés ci-dessus. En outre, les résidus ne dépassent jamais 10 $\mathrm{cm}$ et même rarement 5 . La méthode nous apparaît donc adaptée aux levés de complément nécessités par les évaluations environnementales.

\section{Limitations et perspectives}

N'effectuant pas de compensation globale sur l'ensemble des clichés, si un élément à lever n'est pas vu entièrement sur chaque cliché utilisé et qu'il faut recourir à plusieurs séries de clichés différents, les résidus entre les différentes séries sont susceptibles d'être de signe contraire et donc de provoquer un déplacement important de l'objet restitué. Dans l'expérimentation que nous avons présentée le nombre de clichés était limité car les façades qui nous intéressaient pouvaient être photographiées en une seule fois. Il serait néanmoins opportun dans l'avenir, de réfléchir à une méthode de compensation globale sur l'objet levé lorsque ce dernier ne peut être vu entièrement sur chaque cliché.

Une autre limitation, est que nous n'avons pas envisagé la texturation du modèle car elle n'est pas utile aux études présentées dans cet article. Il serait utile de s'intéresser à cet aspect des choses dans le cadre de développements futurs pour enrichir le potentiel d'utilisation de la méthode.

Une autre perspective est la liaison de notre application avec un logiciel de CAO en vue d'automatiser et d'accélérer l'obtention de volume à intégrer à la maquette 3D du site d'étude. En effet, comme nous l'avons déjà précisé, le temps est un des aspects les plus critiques lors des procédures d'évaluation des incidences des plans et projets. Parallèlement à cela, une intégration des modules DLT au logiciel de 
Article publié dans la Revue Internationale de Géomatique. Volume 18(2) - n 2/2008, pages 147 à 167

photogrammétrie DDPS (Schumacker, 2002) développé au sein d'une de nos équipes de recherche est également envisagée.

Enfin, nous entamons également une réflexion sur la procédure de collecte des données sur le terrain. Comme nous l'avons dit, la DLT 3D impose que les points d'appui utilisés ne soient pas coplanaires. Or, lorsqu'il s'agit de lever des façades de bâtiment, cette contrainte peut parfois devenir importante. Nous réfléchissons dès lors à l'utilisation d'un volume témoin à placer devant les façades et dont la géométrie serait parfaitement connue. L'intérêt d'un tel volume serait multiple :

- Si une seule série de clichés est nécessaire pour lever l'élément d'intérêt et qu'il existe, sur ces clichés, suffisamment de points communs avec le modèle 3D à compléter, en laissant le volume témoin en place entre les clichés, la reconstruction 3D pourrait s'effectuer uniquement sur base du système de coordonnées associé au volume. Une fois, l'élément reconstruit en $3 \mathrm{D}$, il devrait être recalé dans le système du modèle général sur base des points communs. On éliminerait de la sorte la nécessité d'utiliser une station totale sur le terrain.

- Par contre, si plusieurs séries de clichés sont nécessaires, le théodolite restera indispensable, mais l'utilisation du volume témoin limitera à deux le nombre de points à lever par série de clichés (pour orienter une arête du volume).

\section{Conclusions}

Le projet SUIT avait pour but de palier le manque de références en matière de techniques d'évaluation de projets et de plans, et en particulier de l'impact de ces projets ou ces plans sur le patrimoine bâti. Il a permis de développer des méthodes d'évaluation adaptées aux Etudes d'Incidence sur l'Environnement et aux Evaluations Environnementales Stratégiques réalisées dans les centres urbains historiques. Ce type d'étude exige une modélisation 3D adaptée des bâtiments, du mobilier urbain et des réseaux de surface. Nous avons dès lors souhaité proposer une technique de photogrammétrie terrestre, la DLT 3D, afin de compléter les données issues de procédés plus lourds tels que la photogrammétrie aérienne par exemple.

Plus qu'un souci de grande précision, c'est avant tout une exigence de rapidité et d'efficacité dans la mise en œuvre qui dicte l'utilisation des techniques de relevé étant donné le temps et les ressources d'ordinaire disponibles pour la constitution des données de base dans le cadre de ces études. Les tests effectués sur l'utilisation de la technique DLT 3D pour l'acquisition de données tridimensionnelles d'une séquence de bâtiments en site urbain, sont concluants. L'erreur de positionnement 
est tout à fait dans la tolérance que nous nous étions fixée a priori, et la mise en œuvre de la méthode est rapide et simple.

Il nous reste à souligner que la plupart des applications développées dans le cadre du projet SUIT s'inscrivent en phase amont des évaluations environnementales. Elles visent essentiellement à faciliter le repérage d'éléments d'intérêt patrimonial ainsi qu'à évaluer les effets de projets ou de plans sur la cohérence spatiale et fonctionnelle de fragments urbains. Les modèles 3D produits de la sorte permettent de réaliser des simulations informatiques de l'impact d'un projet sur la morphologie urbaine, de proposer des visualisations avant/après réalisation d'un projet ou encore de fournir un support documentaire actualisé des valeurs patrimoniales d'un site.

La technique de photogrammétrie que nous avons présentée ouvre également la voie à un nouveau type d'application. Il s'agit du suivi à long terme des effets d'un plan ou d'un projet sur l'environnement urbain. La récolte et le calibrage de clichés photographiques, à intervalles réguliers et sur de longues périodes de temps, permettrait ainsi de réaliser un suivi détaillé de l'évolution d'un fragment urbain suite à un ensemble d'interventions. Une telle application permettrait de répondre à l'une des exigences de la Directive en matière d'Evaluation Environnementale Stratégique, à savoir de mettre en place des mécanismes de suivi des effets directs ou indirects des plans d'aménagement sur l'environnement. La technique proposée dans le cadre de cet article conviendrait parfaitement à une telle application « aval», en raison de sa souplesse et de sa légèreté.

\section{Bibliographie}

Baltsavias, E.P., Gruen, A., «Resolution convergence - A comparison of aerial photos, LIDAR and IKONOS for monitoring cities », Remotely Sensed Cities, Mesev V. (Ed.), Taylor \& Francis, London, 2003, p. 47-82.

CE (2003), Directive 85/337/EEC du Conseil, sur l'évaluation des effets de certains projets publics et privés sur l'environnement, amendée par les directives 97/11/EC et 2003/35/EC.

CE (2001), Directive 2001/42/EC du Parlement Européen et du Conseil, sur les effets de certains plans et programmes sur l'environnement.

Chehata N., Modélisation $3 D$ de scènes urbaines à partir d'images satellitaires à très haute résolution, Thèse de doctorat de l'Université Paris 5, 2005, 207 p.

Da Col A., Billen R., Schumacker B., Ewiak I., Kaczyński R., «Educational Software for Digital Photogrammetry », Polish Society for Photogrammetry and Remote Sensing biannual Conference, Arch. of Photogrammetry, Cartography and Remote Sensing, Vol. 12b, Varsovie, Pologne, 2002, p. 79 - 84. 
Article publié dans la Revue Internationale de Géomatique. Volume 18(2) - n 2/2008, pages 147 à 167

Drap P., Grussenmeyer P., Arpenteur : an ARchitectural PhotogrammEtric Network Tool for EdUcation and Research. (http://www.arpenteur.net), 2005

Früh C., Zakhor A., « Constructing 3D City Models by merging ground-based and airborne views », IEEE Computer Graphics and Applications, Special Issue Nov/Dec, 2003.

Fuchs F., Jibrini H., Maillet G., Pierrot-Deseilligny M., Taillandier F., « Trois approches pour la construction automatique de modèles 3D de bâtiments en imagerie aérienne haute résolution », Bulletin de la SFPT, 166:pp 10-18, Saint-Mandé, France, 2002.

Glasson J., Therivel R., Chadwick A., Introduction to Environment Impact Assessment, The natural and built environment series, UCL Press, 1994.

Goodey B., "Landscape », Methods of Environmental Impact Assessment, P. Morris et R. Therivel (eds.), UCL Press, 1995, p. 78-95.

Gruen A., Zhang L., Wang X., «3D City Modeling with TLS (Three Line Scanner) Data », International Archives of Photogrammetry, Remote Sensing and Spatial Information Sciences, Vol. XXXIV, Part 5/W10, International Workshop on Visualization and Animation of Reality-based 3D Models, 24-28 February 2003, Tarasp-Vulpera, Switzerland, CD-ROM

Haala N., Brenner C., «Virtual City Models from Laser Altimeter and 2D Map Data », Photogrammetric Engineering and Remote Sensing, Vol. 65, No. 7, July 1999, pp. 787795.

Haala N., «On The Refinement of Urban Models by Terrestrial Data Collection », ISPRS Congress 2004, Volume 35, Part B, Istanbul, Turkey, CD-ROM, 6 pages

Kohler N., « Task 3.3c - Integrated Life Cycle Analysis in the sustainability assessment of urban historical areas », 2003, Rapport SUIT. Disponible sur http://www.suitproject.net/

Kohler N., Bender T., « Task 1.4 - Decision-matrix gathering of existing tools/methodologies applicable during the Environmental Assessment of an historical area active conservation », 2002, Rapport SUIT. Disponible sur http://www.suitproject.net/

Kim Z., Huertas A., Nevatia R., « Automatic description of buildings with complex rooftops from multiple images », Computer Vision and Pattern Recognition, Kauai, 2001, p. 272279.

Kwon Y.-H., The DLT Method, Site Internet de Kwon3D, 2003, (http://kwon3d.com/theory/dlt/dlt.html)

Land Use Consultants, EIA Costs and benefits Study, Directorat Général - Environnement, 1996.

Laplanche F., «Spécification d'une méthodologie de relevés adaptée aux enquêtes environnementales stratégiques en milieu urbain» Mémoire de DES en urbanisme et aménagement du territoire », Faculté des Sciences Appliquées, Université de Liège. Inédit. 2004. 96 p.

Morris P., Therivel R., Methods of Environmental Impact Assessment, UCL Press, 1995.

Photomodeler, Photomodeler: Measuring and modeling the real world, 2005, (http://www.photomodeler.com) 
PICC, Le Projet Informatique de Cartographie Continue. Site de la Direction de la Topographie et de la Cartographie du Ministère de l'Equipement et des Transports, 2004, (http://internet.win.be/rainbow/d432/picc.htm)

Rottensteiner F., Trinder J., Clode S., Kubik K., « Detection of buildings and roof segments by combining LIDAR data and multispectral images », Image and Vision Computing, New Zealand, 2003, p. 60-65.

Rottensteiner, F., Jansa, J., « Automatic Extraction of Building from LIDAR Data and Aerial Images », IAPRS, Vol.34, Part 4, 2002, p. 295-301.

Stamos I., Allen P., « 3-D Model Construction Using Range and Image Data ». CVPR, 2000.

Schumacker B., DDPS - Digital \& Didactic Photogrammetric Software, Site Internet, 2002, (http://www.geo.ulg.ac.be/ddps).

Teller J., «A spherical metric for the field-oriented analysis of complex urban open spaces », Environment and Planning B: Planning and Design, 30(3), 2003, p. 339-356.

Therivel R., Wilson E., Thompson S., Heaney D., Pritchard D., Strategic Environmental Assessment, Earthscan, 1992, Londres.

Tweed C., Sutherland M., Teller J., Visualisation Tools To Aid Public Understanding of New Developments in Urban Historical Areas, Rapport SUIT, 2003, Disponible sur http://www.suitproject.net/

Wiechert A., « Linking Laser Scanning to Surveying an Visualization - Integrating Geotechnologies with a New Beaming Method». GeoInformatics, Vol 7, n5, july/August 2004, p. 48-51.

Zhao H., Shibasaki R., «A Robust Method for Registering Ground-Based Laser Range Images of Urban Outdoor Objects », Photogrammetric Engineering and Remote Sensing, Vol. 67, No 10, 2001a, pp. 1143-1153.

Zhao H., Shibasaki R., «Reconstructing Urban 3D Model using Vehicle-borne Laser Range Scanners », 3-D Digital Imaging and Modeling, Proceedings, Quebec, du 28/5 au 1/6/2001, 2001 b (http://shiba.iis.u-tokyo.ac.jp/member/current/zhao/pub/3dim2001.pdf) 